\title{
Analysis of Equation of States for the Suitability at High Pressure: MgO as an Example
}

\author{
Kuldeep Kholiya, ${ }^{1}$ Jeewan Chandra, ${ }^{2}$ and Swati Verma ${ }^{3}$ \\ ${ }^{1}$ Department of Applied Science, B.T. Kumaon Institute of Technology, Dwarahat 263653, India \\ ${ }^{2}$ Department of Applied Science, G.B. Pant Engineering College Ghurdauri, Pauri Garhwal 246194, India \\ ${ }^{3}$ Department of Computer Science, B.T. Kumaon Institute of Technology, Dwarahat 263653, India
}

Correspondence should be addressed to Kuldeep Kholiya; kuldeep_phy1@rediffmail.com

Received 31 August 2013; Accepted 22 October 2013; Published 16 January 2014

Academic Editors: V. Chaldyshev and L. Sheng

Copyright (C) 2014 Kuldeep Kholiya et al. This is an open access article distributed under the Creative Commons Attribution License, which permits unrestricted use, distribution, and reproduction in any medium, provided the original work is properly cited.

\begin{abstract}
A simple theoretical model is developed to study the high pressure behavior of solids and is applied to evaluate the pressure for $\mathrm{MgO}$ in case of large compression along with Shanker, Tait, Vinet, and Birch-Murnaghan equation of states (EOSs). These EOSs are also tested for the basic requirements revealed from the fundamental thermodynamics for solids in the limit of extreme compressions, as given by Stacey. It is found that for the high pressure compression behavior of $\mathrm{MgO}$ the present model, Tait, Vinet, and BirchMurnaghan EOSs give the results compatible with the experimental data. It has also been found that in the regime of ultrahigh pressure the present model and Birch-Murnaghan EOS satisfy the Stacey criterion.
\end{abstract}

\section{Introduction}

Strength and elastic properties of a solid depend on the strength of its interatomic forces. Therefore, the application of pressure which changes interatomic distance of the substances changes its physical properties. This pressure versus volume relation is known as equation of state and may be quite useful to understand the physical properties of the material. In the literature, there are a number of equations of state, and these arise from an unchecked and unverifiable assumption concerning an assumed interatomic potential, an assumed strain function, or an assumed boundary condition that cannot be tested [1]. Therefore, the fitting of the experimental pressure-volume data has been the usual practice to prove the suitability of an EOS in different class of solids. However, the fitting of $P-V$ data up to the small pressure range does not provide any support for the validity of the EOS beyond the fitting range due to two reasons.

(i) For small compression almost every EOS gives the results within the experimental uncertainty, as laboratory measured $P-V$ data are often subjected to pressure calibration errors [2]. (ii) Almost every EOS, new or old, can be fitted to a given range of $P-V$ data by adjusting the values of the zero pressure, isothermal bulk modulus $\left(B_{0}\right)$, and its pressure derivatives $\left(B_{0}^{\prime}, B_{0}^{\prime \prime}\right)[2]$.

Thus, the aim of the present work is to discuss the applicability of Shanker [3], Tait $[4,5]$, Vinet [6], and BirchMurnaghan EOSs [7] along with an equation of state recently formulated by Kholiya [8] as well as to check the validity of these EOSs for Stacey criterion under infinite pressure. For this purpose $\mathrm{MgO}$ is taken as an example due to the reasons that the phase transition pressure for it is greater than $300 \mathrm{GPa}[9,10]$ and a large number of experimental and theoretical studies [9-33] have done for it so a large number of data is available up to the very large pressure for the sake of comparison.

\section{Method of Analysis}

On the basis of the findings that the repulsive branch of binding energy curves can be represented by a simple function 
of density Parsafar and Mason [34] have considered an EOS as

$$
P\left(\frac{V}{V_{0}}\right)^{2}=A_{0}+A_{1}\left(\frac{V}{V_{0}}\right)^{-1}+A_{2}\left(\frac{V}{V_{0}}\right)^{-2},
$$

where $V / V_{0}=\rho_{0} / \rho$, and $V_{0}, \rho_{0}$ are the zero pressure values of volume $V$ and density $\rho$, respectively, and $A_{0}, A_{1}$, and $A_{2}$ are constants at a particular temperature. On the basis of the first-principle calculations, using the augmented-plane-wave (APW) method and quantum statistical model Hama and Suito [35] revealed that the Parsafar-Mason EOS becomes less successful at high compressions $\left(V / V_{0}<0.65\right)$. In addition to this, Shanker and Kushwah [36] pointed out that the Parsafar and Mason EOS gives $P$ as a fourth degree expression in $V / V_{0}$; therefore, the determination of the higher-derivative properties such as the bulk modulus and its pressure derivatives becomes less convenient. Shanker and Kushwah [36] have expanded $P V^{2}$ in powers of $\left[1-\left(V / V_{0}\right)\right]$ up to the quadratic term and found that the equation works well for materials having pressure derivative of the isothermal bulk modulus less than four. Recently, Kholiya [8] has expanded pressure in powers of density up to the quadratic term and achieved the EOS as

$$
\begin{aligned}
& P\left(V, T_{0}\right) \\
& =\frac{B_{0}}{2}\left[\left(B_{0}^{\prime}-3\right)-2\left(B_{0}^{\prime}-2\right)\left(\frac{V}{V_{0}}\right)^{-1}+\left(B_{0}^{\prime}-1\right)\left(\frac{V}{V_{0}}\right)^{-2}\right] .
\end{aligned}
$$

In the present study we have also considered Shanker, Tait, Vinet, and Birch-Murnaghan equation of states for the comparison purpose. These EOS may be given as [3-7]:

$$
\begin{gathered}
P\left(V, T_{0}\right)=B_{0}\left[\left(1-\frac{V}{V_{0}}\right)+\left(\frac{B_{0}^{\prime}+1}{2}\right)\left(1-\frac{V}{V_{0}}\right)^{2}\right], \\
P\left(V, T_{0}\right)=\frac{B_{0}}{B_{0}^{\prime}+1}\left[\exp \left\{\left(B_{0}^{\prime}+1\right)\left(1-\frac{V}{V_{0}}\right)\right\}-1\right] \\
P=3 B_{0}\left(\frac{V}{V_{0}}\right)^{-2 / 3}\left[1-\left(\frac{V}{V_{0}}\right)^{1 / 3}\right] \exp \left[X\left\{1-\left(\frac{V}{V_{0}}\right)^{1 / 3}\right\}\right] \\
P=\frac{3}{2} B_{0}\left[\left(\frac{V_{0}}{V}\right)^{7 / 3}-\left(\frac{V_{0}}{V}\right)^{5 / 3}\right] \\
\times\left[1+\frac{3}{4}\left(B_{0}^{\prime}-4\right)\left\{\left(\frac{V_{0}}{V}\right)^{2 / 3}-1\right\}\right] .
\end{gathered}
$$

Equations (3), (4), (5), and (6) are Shanker, Tait, Vinet and Birch-Murnaghan equation of states, respectively, and here $B_{0}$ and $B_{0}^{\prime}$ are the bulk modulus and its first order pressure derivative at $P=0$ and $T=T_{0}$, respectively, and $X=(3 / 2)\left(B_{0}^{\prime}-\right.$ $1)$.
The bulk modulus may be given as, $B=-V(d P / d V)$ so the expression for bulk modulus from (2)-(6) comes out as

$$
\begin{gathered}
B=B_{0}\left(B_{0}^{\prime}-2\right)\left(\frac{V}{V_{0}}\right)^{-1}\left[\left(\frac{B_{0}^{\prime}-1}{B_{0}^{\prime}-2}\right)\left(\frac{V}{V_{0}}\right)^{-1}-1\right], \\
B=B_{0}\left(\frac{V}{V_{0}}\right)\left[1+\left(B_{0}^{\prime}+1\right)\left\{1-\frac{V}{V_{0}}\right\}\right], \\
B=B_{0}\left(\frac{V}{V_{0}}\right)\left[\exp \left\{\left(B_{0}^{\prime}+1\right)\left(1-\frac{V}{V_{0}}\right)\right\}\right], \\
B=B_{0}\left(\frac{V}{V_{0}}\right)^{-2 / 3}\left[1+\left\{X\left(\frac{V}{V_{0}}\right)^{1 / 3}+1\right\}\left\{1-\left(\frac{V}{V_{0}}\right)^{1 / 3}\right\}\right] \\
\times \exp \left[X\left\{1-\left(\frac{V}{V_{0}}\right)^{1 / 3}\right\}\right], \\
B=\frac{1}{2} B_{0}\left[7\left(\frac{V_{0}}{V}\right)^{7 / 3}-5\left(\frac{V_{0}}{V}\right)^{5 / 3}\right] \\
+\frac{3}{8} B_{0}\left(B_{0}^{\prime}-4\right)\left[9\left(\frac{V_{0}}{V}\right)^{3}-14\left(\frac{V_{0}}{V}\right)^{7 / 3}+5\left(\frac{V_{0}}{V}\right)^{5 / 3}\right] .
\end{gathered}
$$

The corresponding expressions for the first order pressure derivative of bulk modulus $B^{\prime}=d B / d P$ obtained from (7) comes out as

$$
\begin{gathered}
B^{\prime}=\frac{\left(B_{0}^{\prime}-2\right)\left(V / V_{0}\right)-2\left(B_{0}^{\prime}-1\right)}{\left(B_{0}^{\prime}-2\right)\left(V / V_{0}\right)-\left(B_{0}^{\prime}-1\right)} \\
B^{\prime}=\frac{\left(B_{0}^{\prime}+1\right)\left(2 V / V_{0}-1\right)-1}{\left(B_{0}^{\prime}+1\right)\left(1-\left(V / V_{0}\right)\right)+1} \\
B^{\prime}=\left(\frac{V}{V_{0}}\right)\left(B_{0}^{\prime}+1\right)-1, \\
B^{\prime}=\frac{1}{3}\left[\frac{\left(V / V_{0}\right)^{1 / 3}(1-X)+2 X\left(V / V_{0}\right)^{2 / 3}}{1+\left\{X\left(V / V_{0}\right)^{1 / 3}+1\right\}\left\{1-\left(V / V_{0}\right)^{1 / 3}\right\}}\right. \\
\left.+X\left(\frac{V}{V_{0}}\right)^{1 / 3}+2\right], \\
B^{\prime}=\frac{B_{0}}{8 B}\left[\left(B_{0}^{\prime}-4\right)\left\{81\left(\frac{V_{0}}{V}\right)^{3}-98\left(\frac{V_{0}}{V}\right)^{7 / 3}+25\left(\frac{V_{0}}{V}\right)^{5 / 3}\right\}\right. \\
\left.+\frac{4}{3}\left\{49\left(\frac{V_{0}}{V}\right)^{7 / 3}-25\left(\frac{V_{0}}{V}\right)^{5 / 3}\right\}\right] .
\end{gathered}
$$

All these EOSs are also tested for the basic criteria which must be satisfied by an EOS for its validity and applicability as suggested by Stacey $[2,37,38]$. These criteria are as follows.

(i) In the limit $\rightarrow \infty, V / V_{0} \rightarrow 0$.

(ii) With the increase in pressure isothermal bulk modulus must increase continuously and in the limit of infinite pressure $B \rightarrow \infty$. 


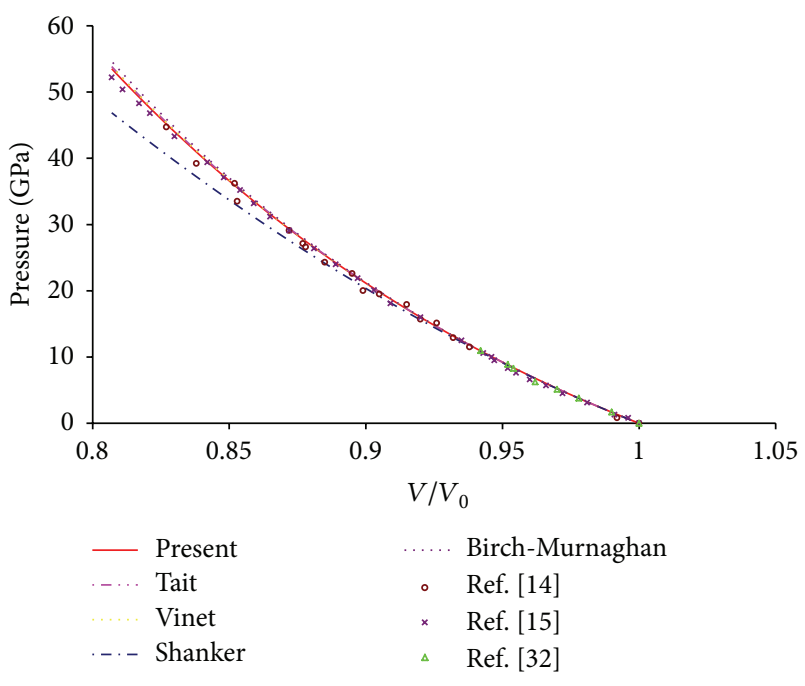

Figure 1: Compression behavior of MgO using different EOSs along with the experimental results.

(iii) $B^{\prime}$ must decrease progressively with the increase in pressure such that $B^{\prime}$ remains greater than $5 / 3$ in the limit of infinite pressure.

\section{Results and Discussions}

To study the compression behavior the present study requires two input parameters, namely, $B_{0}, B_{0}^{\prime}$. In the literature a lot of experimental values of $B_{0}$ and $B_{0}^{\prime}$ are available for $\mathrm{MgO}$ for example, $B_{0}=160$ [13], 161 [14], 160.2 [15], 161.4 [28], 160.2 [29], 153 [30], 160.2 [31], 161.3 [32] and $B_{0}^{\prime}=4.15$ [13], 3.94 [14], 3.99 [15], 4.29 [28], 4.15 [29], 4.1 [30], 4.03 [31], 4.24 [32]. In the low compression range by choosing the suitable set of input parameters all the equations can be fitted to give the experimental results as at low pressure all EOSs give almost the same results. But as pressure increases the difference of the values calculated from different EOSs increase. For high compression the small difference in $B_{0}$ and $B_{0}^{\prime}$ has little effect on the calculated values and at high compression the form of an EOS becomes more significant and different EOSs give different results.

In the present study the values of $B_{0}$ and $B_{0}^{\prime}$ are taken to be 161.3 GPa and 4.24 as obtained by Li et al. [32], who measured the bulk modulus and its first order pressure derivative by fitting the experimentally measured velocity and density data to the third-order finite strain equations (Birch-Murnaghan EOS). The reason for this selection is that these values are the more recent values. The values of pressure calculated from (2) (present model), (3) (Shanker EOS), (4) (Tait EOS), (5) (Vinet EOS), and (6) (Birch-Murnaghan EOS) at different compression are plotted in Figure 1 along with the experimental findings. From Figure 1 it is clear that (2) (present model), (4) (Tait EOS), (5) (Vinet EOS), and (6) (Birch-Murnaghan EOS) give results compatible with the experimental findings while (3) (Shanker EOS) gives low results and hence fails at high pressure. It is pertinent to mention here that the selection of other set of input parameters also provides analogous outcome. It is important to mention here that for all the form

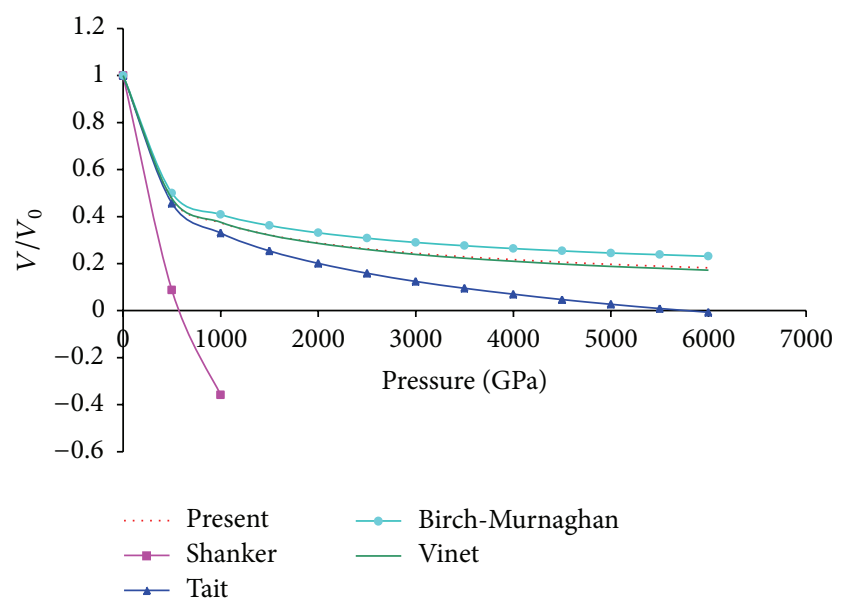

FIGURE 2: Compression behavior at ultrahigh pressure using different EOSs.

of the EOSs value of $B_{0}$ and $B_{0}^{\prime}$ is the same (161.3 GPa and 4.24) and the experimental values are taken from the respective papers. These EOS are also tested for the Stacey criteria arise from basic thermodynamic conditions. Figure 2 shows the variation of $V / V_{0}$ with pressure and from this figure it is clear that for Shanker and Tait EOSs $V / V_{0}$ become zero at a finite value of pressure while for present, Vinet and Birch-Murnaghan $\operatorname{EOS} V / V_{0}$ becomes zero only in the limit of infinite pressure. At ultrahigh pressure it may also be noted that for compression the present model and Vinet EOS give almost similar results while Birch-Murnaghan EOS gives slightly higher results in comparison to these two EOSs. Here, it may be argued in favor of Shanker and Tait EOS that if any conventional solid were to approach infinite compression it would undergo dramatic phase transitions to exotic forms and equations of state do not carry through phase transitions but an equation of state must satisfy basic physical laws, in particular thermodynamic relationships; even outside the pressure ranges over which the materials that it describes can exist in the same phase because phase transition only changes the values of the parameters and not the basic thermodynamic relationship. The variation of isothermal bulk modulus $B$ with pressure for $\mathrm{MgO}$ by using present, Shanker, Tait, Vinet, and Birch-Murnaghan EOSs is plotted in Figure 3. From Figure 3 it can be revealed that for Shanker and Tait EOSs initially $B$ increases then decreases and finally becomes negative with the increase in pressure while for present Vinet and BirchMurnaghan EOSs with the increase in pressure isothermal bulk modulus increases continuously and sharply so that it approaches to infinity in the limit of infinite pressure. At higher compression this continuous and sharp increase in bulk modulus may be attributed to the fact that at larger compressions the repulsive forces become so large. Figure 4 represents the variation of first order pressure derivative of bulk modulus $B^{\prime}$ with pressure. Again from this figure it is clear that for Shanker and Tait EOSs at high pressure $B^{\prime}$ becomes negative while for present Vinet and Birch-Murnaghan EOSs it decreases progressively with the increase in pressure and then becomes asymptotic. From (8), (11), and (12) it may 


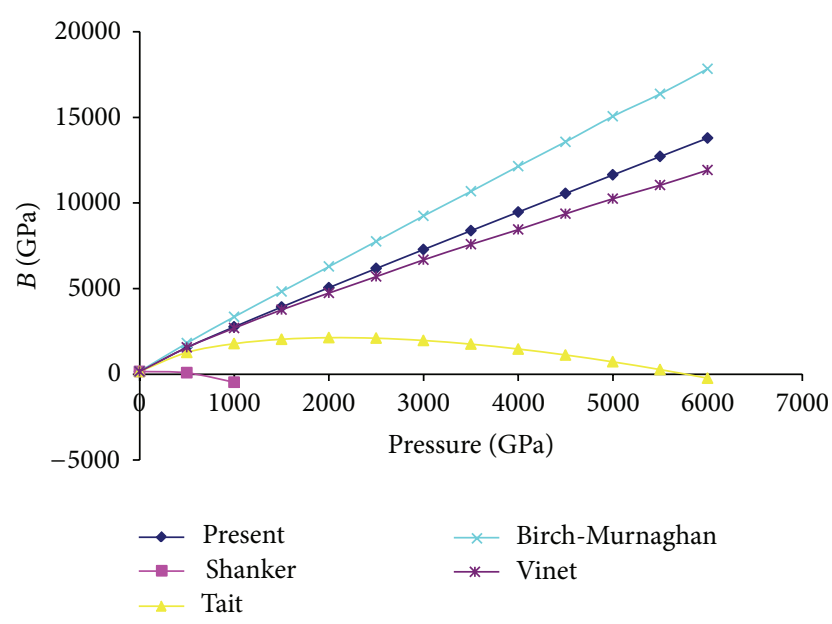

FIGURE 3: High pressure behavior of bulk modulus using different EOSs.

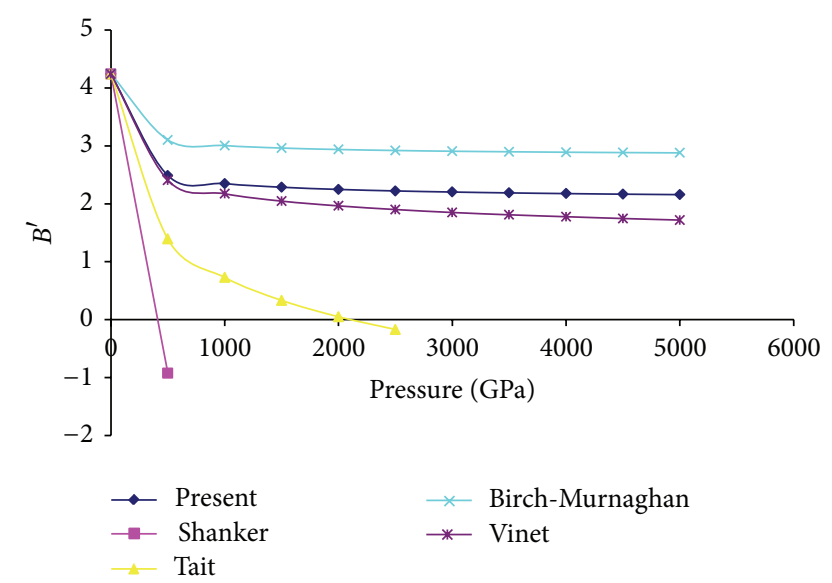

FIGURE 4: High pressure behavior of first order pressure derivative of bulk modulus using different EOSs.

be noted that for present Vinet and Birch-Murnaghan EOSs the value of $B_{\infty}^{\prime}$ (first order pressure derivative of bulk modulus at infinite pressure) comes out to be $2,2 / 3$, and 3 , respectively. Therefore, Vinet does not follow the third criteria of the Stacey's basic criterion of an EOS that is, in the limit of infinite pressure $B^{\prime}$ should remain greater than $5 / 3$ while the present model and Birch-Murnaghan EOS follow all the three Stacey's criteria of an EOS.

\section{Conclusions}

From the overall discussion it may be concluded that the present formulation which was developed for nanomaterials [8] by expanding pressure in powers of density up to the quadratic term is also valid for the bulk materials. The formulation not only reproduces the experimental results regarding compression of bulk $\mathrm{MgO}$ but also satisfies the criteria based on the basic thermodynamic relations. Tait, Vinet, and BirchMurnaghan EOSs give the results compatible with the experimental findings but Tait and Vinet EOSs fail to satisfy the basic criteria of an EOS. While at higher compression
Shanker EOS does not give the satisfactory results and also fails when checked for the basic criteria of an EOS. Although, the Birch-Murnaghan EOS follows all the three criteria of an EOS, its shortcomings under very high pressure have been previously pointed out by Stacey [39]. Hence, the present formulation may be quite useful for studying the high pressure elastic behavior of solids.

\section{Conflict of Interests}

The authors declare that there is no conflict of interests regarding the publication of this paper.

\section{References}

[1] O. L. Anderson, Equations of State of Solids for Geophysics and Ceramic Science, Oxford University Press, Oxford, UK, 1994.

[2] F. D. Stacey and P. M. Davis, "High pressure equations of state with applications to the lower mantle and core," Physics of the Earth and Planetary Interiors, vol. 142, no. 3-4, pp. 137-184, 2004.

[3] J. Shanker, S. S. Kushwah, and P. Kumar, "Theory of thermal expansivity and bulk modulus for $\mathrm{MgO}$ and other minerals at high temperatures," Physica B, vol. 233, no. 1, pp. 78-83, 1997.

[4] J. R. MacDonald, "Review of some experimental and analytical equations of state," Reviews of Modern Physics, vol. 41, no. 2, pp. 316-349, 1969.

[5] K. Devlal and K. Kholiya, "Analysis of equations of state for metals and rare gas solids," Indian Journal of Physics, vol. 80, no. 8, pp. 801-805, 2006.

[6] P. Vinet, J. H. Rose, J. Ferrante, and J. R. Smith, "Universal features of the equation of state of solids," Journal of Physics, vol. 1, no. 11, article 002, pp. 1941-1963, 1989.

[7] F. Birch, "Elasticity and constitution of the Earth's interior," Journal of Geophysical Research, vol. 57, no. 2, pp. 227-286, 1952.

[8] K. Kholiya, "High pressure isothermal equation of state for nanomaterials," Indian Journal of Physics, vol. 87, no. 4, pp. 339-343, 2013.

[9] P. I. Dorogokupets and A. Dewaele, "Equations of state of MgO, $\mathrm{Au}, \mathrm{Pt}, \mathrm{NaCl}-\mathrm{B} 1$, and NaCl-B2: internally consistent high-temperature pressure scales," High Pressure Research, vol. 27, no. 4, pp. 431-446, 2007.

[10] P. I. Dorogokupets and A. R. Oganov, "Ruby, metals, and MgO as alternative pressure scales: a semiempirical description of shock-wave, ultrasonic, $\mathrm{x}$-ray, and thermochemical data at high temperatures and pressures," Physical Review B, vol. 75, no. 2, Article ID 024115, 2007.

[11] T. S. Duffy, R. J. Hemley, and H.-K. Mao, "Equation of state and shear strength at multimegabar pressures: magnesium oxide to 227 GPa," Physical Review Letters, vol. 74, no. 8, pp. 1371-1374, 1995.

[12] H. K. Mao and P. M. Bell, "Equations of state of Mg0 and xi Fe under static pressure conditions," Journal of Geophysical Research, vol. 84, no. 9, pp. 4533-4536, 1979.

[13] Y. Fei, "Effects of temperature and composition on the bulk modulus of (Mg,Fe)O," American Mineralogist, vol. 84, no. 3, pp. 272-276, 1999.

[14] A. Dewaele, G. Fiquet, D. Andrault, and D. Hausermann, "P-V-T equation of state of periclase from synchrotron radiation measurements," Journal of Geophysical Research B, vol. 105, no. 2, pp. 2869-2877, 2000. 
[15] S. Speziale, C.-S. Zha, T. S. Duffy, R. J. Hemley, and H.-K. Mao, "Quasi-hydrostatic compression of magnesium oxide to $52 \mathrm{GPa}$ : implications for the pressure-volume-temperature equation of state," Journal of Geophysical Research B, vol. 106, no. 1, pp. 515528, 2001.

[16] S. D. Jacobsen, C. M. Holl, K. A. Adams et al., "Compression of single-crystal magnesium oxide to $118 \mathrm{GPa}$ and a ruby pressure gauge for helium pressure media," American Mineralogist, vol. 93, no. 11-12, pp. 1823-1828, 2008.

[17] K. Hirose, N. Sata, T. Komabayashi, and Y. Ohishi, "Simultaneous volume measurements of $\mathrm{Au}$ and $\mathrm{MgO}$ to $140 \mathrm{GPa}$ and thermal equation of state of Au based on the MgO pressure scale," Physics of the Earth and Planetary Interiors, vol. 167, no. 3-4, pp. 149-154, 2008.

[18] O. L. Anderson and K. Zou, "Thermodynamic functions and properties of $\mathrm{MgO}$ at high compression and high temperature," Journal of Physical and Chemical Reference Data, vol. 19, no. 1, article 69, 1990.

[19] J. Hama and K. Suito, "Thermoelastic properties of periclase and magnesiowüstite under high pressure and high temperature," Physics of the Earth and Planetary Interiors, vol. 114, no. 3-4, pp. 165-179, 1999.

[20] M. Matsui, S. C. Parker, and M. Leslie, "The MD simulation of the equation of state of $\mathrm{MgO}$ : application as a pressure calibration standard at high temperature and high pressure," American Mineralogist, vol. 85, no. 2, pp. 312-316, 2000.

[21] B. B. Karki, R. M. Wentzcovitch, S. de Gironcoli, and S. Baroni, "High-pressure lattice dynamics and thermoelasticity of $\mathrm{MgO}$," Physical Review B, vol. 61, no. 13, pp. 8793-8800, 2000.

[22] A. R. Oganov and P. I. Dorogokupets, "All-electron and pseudopotential study of MgO: equation of state, anharmonicity, and stability," Physical Review B, vol. 67, no. 22, Article ID 224110, 2003.

[23] Y. Wang, Z.-K. Liu, L.-Q. Chen, L. Burakovsky, and R. Ahuja, "First-principles calculations on MgO: phonon theory versus mean-field potential approach," Journal of Applied Physics, vol. 100, no. 2, Article ID 023533, 2006.

[24] Y. Tange, Y. Nishihara, and T. Tsuchiya, "Unified analyses for P$\mathrm{V}$-T equation of state of MgO: a solution for pressure-scale problems in high P-T experiments," Journal of Geophysical Research, vol. 114, no. 3, Article ID B03208, 2009.

[25] Z. Q. Wu, R. M. Wentzcovitch, K. Umemoto, B. S. Li, K. Hirose, and J. C. Zheng, "Pressure-volume-temperature relations in MgO: an ultrahigh pressure-temperature scale for planetary sciences applications," Journal of Geophysical Research, vol. 113, no. 6, Article ID B06204, 2008.

[26] K. Jin, Q. Wu, F. Q. Jing, and X. Z. Li, "Simple method for reducing shock-wave equation of state to zero Kelvin isotherm for metals," Journal of Applied Physics, vol. 105, no. 4, Article ID 043510, 2009.

[27] K. Jin, X. Li, Q. Wu et al., "The pressure-volume-temperature equation of state of $\mathrm{MgO}$ derived from shock Hugoniot data and its application as a pressure scale," Journal of Applied Physics, vol. 107, no. 11, Article ID 113518, 2010.

[28] M. S. Vassiliou and T. J. Ahrens, "Hugoniot equation of state of periclase to $200 \mathrm{GPa}$," Geophysical Research Letters, vol. 8, no. 7, pp. 729-732, 1981.

[29] I. Jackson and H. Neisler, High-Pressure Research: Application to Earth and Planetary Sciences, vol. 67 of Geophysical Monograph Series, Edited by S. Akimoto, M. H. Manghnani, Terra Scientific, Tokyo, Japan, 1982.
[30] W. Utsumi, D. J. Weidner, and R. C. Lieberman, High-Pressure Research: Application to Earth and Planetary Sciences, vol. 101 of Geophysical Monograph Series, Edited by M.H. Manghnani, T. Yagi, AGU, Washington, DC, USA, 1998.

[31] C.-S. Zha, H.-K. Mao, and R. J. Hemley, "Elasticity of MgO and a primary pressure to $55 \mathrm{GPa}$," Proceedings of the National Academy of Sciences of the United States of America, vol. 97, no. 25, pp. 13494-13499, 2000.

[32] B. Li, K. Woody, and J. Kung, "Elasticity of MgO to $11 \mathrm{GPa}$ with an independent absolute pressure scale: implications for pressure calibration," Journal of Geophysical Research, vol. 111, no. 11, Article ID B11206, 2006.

[33] P. K. Singh, "Analysis of high derivative thermoelastic properties of MgO," Indian Journal of Physics, vol. 86, no. 4, pp. 259-265, 2012.

[34] G. Parsafar and E. A. Mason, "Universal equation of state for compressed solids," Physical Review B, vol. 49, no. 5, pp. 30493060, 1994.

[35] J. Hama and K. Suito, "The search for a universal equation of state correct up to very high pressures," Journal of Physics Condensed Matter, vol. 8, no. 1, pp. 67-81, 1996.

[36] J. Shanker and S. S. Kushwah, "High-pressure isothermal equation of state for materials with pressure derivative of the isothermal bulk modulus less than four," High Temperatures High Pressures, vol. 33, no. 2, pp. 207-212, 2001.

[37] F. D. Stacey, "The K-primed approach to high-pressure equations of state," Geophysical Journal International, vol. 143, no. 3, pp. 621-628, 2000.

[38] F. D. Stacey, "Finite strain, thermodynamics and the earth's core," Physics of the Earth and Planetary Interiors, vol. 128, no. 1-4, pp. 179-193, 2001.

[39] F. D. Stacey, "Theory of thermal and elastic properties of the lower mantle and core," Physics of the Earth and Planetary Interiors, vol. 89, no. 3-4, pp. 219-245, 1995. 

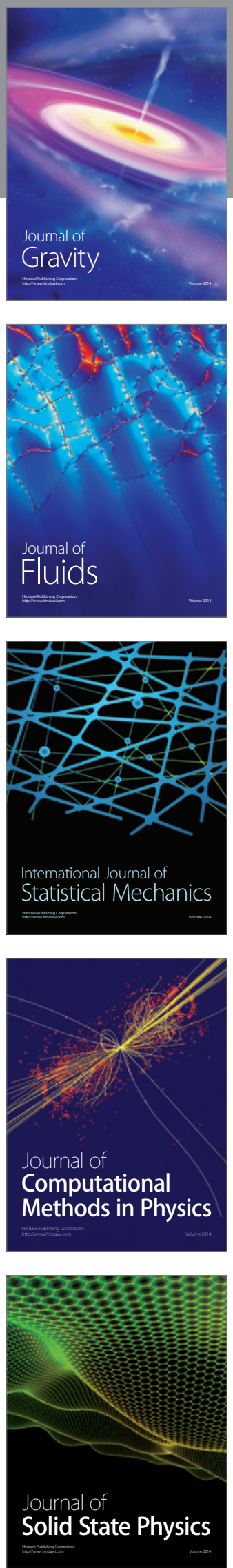

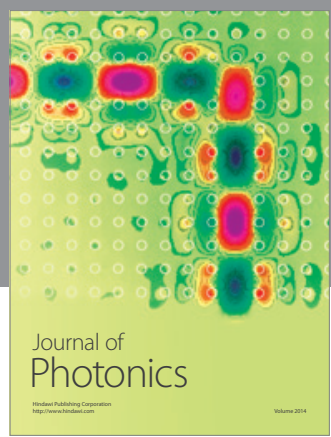

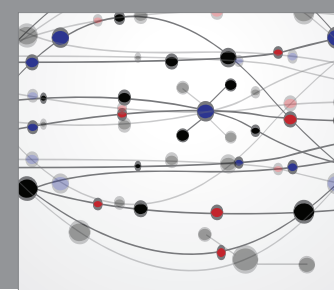

The Scientific World Journal

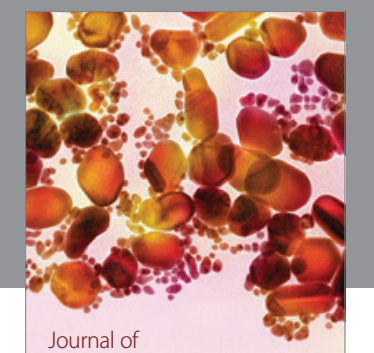

Soft Matter
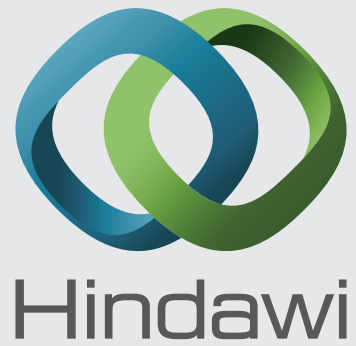

Submit your manuscripts at

http://www.hindawi.com
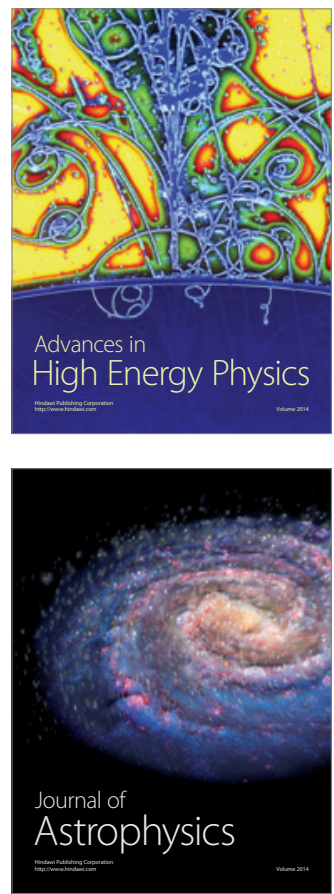
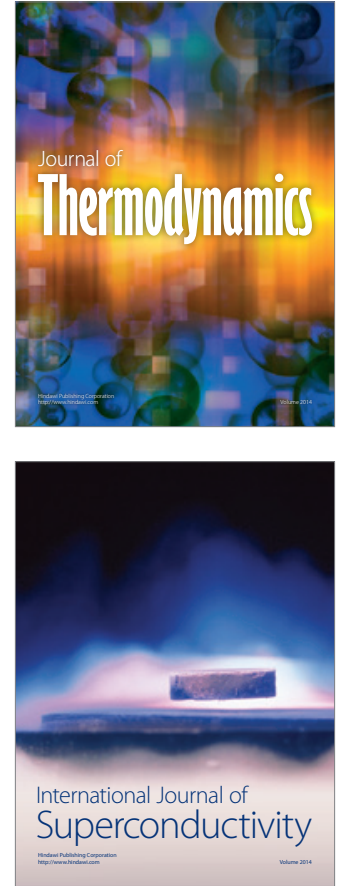
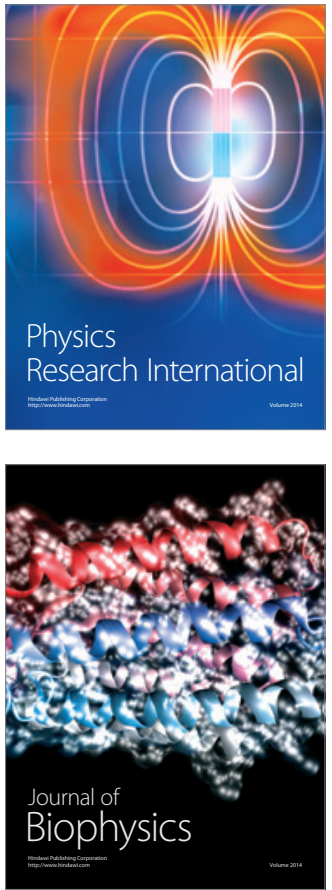
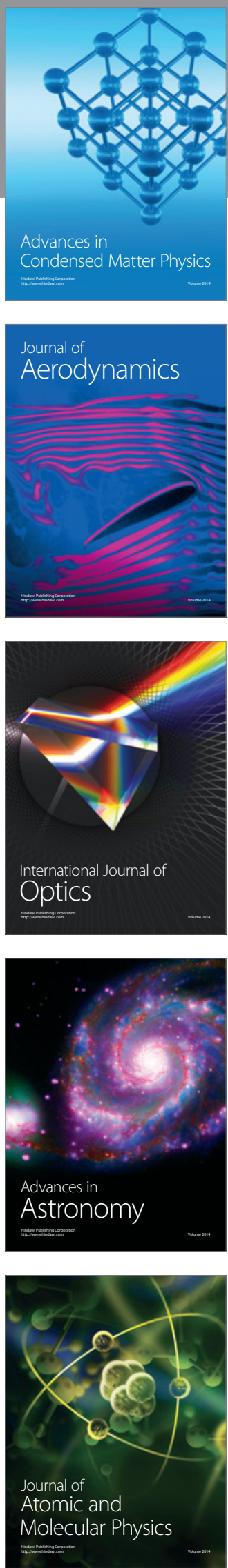